\title{
The Process of How Elderly Patients with Lung Cancer Who Are Receiving Molecularly Targeted Therapy with Oral Agents Establish Self-Management
}

\author{
Akiko Fukawa \\ Faculty of Nursing, Osaka Medical College, Osaka, Japan \\ Email: fon163@osaka-med.ac.jp
}

How to cite this paper: Fukawa, A. (2017) The Process of How Elderly Patients with Lung Cancer Who Are Receiving Molecularly Targeted Therapy with Oral Agents Establish Self-Management. Health, 9, 18011816.

https://doi.org/10.4236/health.2017.913131

Received: November 20, 2017

Accepted: December 12, 2017

Published: December 15, 2017

Copyright $\odot 2017$ by author and Scientific Research Publishing Inc.

This work is licensed under the Creative

Commons Attribution International

License (CC BY 4.0).

http://creativecommons.org/licenses/by/4.0/

\section{(c) (i) Open Access}

\begin{abstract}
Purpose: In Japan, many elderly cancer patients are receiving chemotherapy using oral molecularly targeted drugs. They receive treatment in outpatient setting and have a need to self-manage at home. The purpose of this study was to clarify how elderly patients with lung cancer who are undergoing treatment with molecularly targeted drugs in outpatient setting establish self-management. Methods: The study used Kinoshita's Modified Grounded Theory. Semi-structured interviews were conducted with 17 patients (eight males and nine females). Results: This study identified six categories: Accepting life with cancer, Maintaining activities of daily living without feeling shackled by cancer, Reconsidering how to continue being themselves in daily life until life comes to an end, Using trial and error to integrate treatment and daily living, Formulating their criteria for continuing treatment, and $\mathrm{Ob}$ taining help from caregivers. Conclusion: The self-management process in elderly patients consisted of maintaining treatment and integrating treatment with daily living. And they are exploring the effects of treatment and side effects in order to live their own life. Implications for Nursing: In many elderly patients with lung cancer the purpose of treatment is curative extension of survival and improved quality of life. Our findings suggest that is important we help patients to identify how patients want to live, identify ways to improve their quality of life, and share the goals of treatment between the patient and the caregiver.
\end{abstract}

\section{Keywords}

Elderly Patients, Lung Cancer, Self-Management, Molecularly Targeted Drugs 


\section{Background}

The Japanese population has aged rapidly over recent years, and lung cancer is most prevalent among elderly people [1]. The guideline for diagnosis and treatment of lung cancer [2] recommends the use of molecularly targeted therapy even in elderly patients if they have a specific gene mutation which can be targeted by this form of therapy. Molecularly targeted therapy not only prolongs survival of lung cancer patients with the specific gene mutation but also alleviates symptoms including cough, dyspnea, and pain [3]. However, molecularly targeted therapy is associated with side effects such as rash, diarrhea, and interstitial pneumonia that are complex and unique to this form of therapy. According to a survey on the status of molecularly targeted therapy of lung cancer with oral agents [4], side effects in elderly patients with lung cancer are difficult to manage, and easily lead to worsening of their overall condition. In addition, elderly patients are at a higher risk of developing interstitial pneumonia [5], so that side effects are likely to become life-threatening. Therapy with oral anticancer drugs is generally provided in the outpatient setting, so that patients have to cope with various issues that arise during therapy, including side effects and anxiety concerning disease progression. While elderly patients with lung cancer receive molecularly targeted therapy with oral agents usually in the outpatient setting, only very limited nursing care is available for outpatients on oral anticancer therapy [6]. The main purpose of therapy for cancer patients of advanced age is to maintain their quality of life (QOL) [7], and for this, nurses must provide support to promote self-management tailored specifically to the needs of elderly patients, and help them spend time recuperating as comfortably as possible.

A literature review of the QOL of elderly cancer patients on chemotherapy [8] showed that their QOL is negatively impacted by various side effects that some of these affect elderly patients to a greater extent than younger adult patients, and that comorbidity and activities of daily living (ADL) are likely to influence the QOL of elderly patients. These patients require support across a broad range of psychological and social needs, but at the same time, they demonstrate certain strengths that appear to be unique to this age group. Therefore, nurses should support them in their symptom management in a manner appropriate to the characteristics of elderly patients and help them in their self-management based on a comprehensive understanding of their psychological and social backgrounds. In view of the increasing use of cancer pharmacotherapy in the outpatient setting and progress in the development of new molecularly targeted therapies and oral anticancer agents, the number of patients receiving molecularly targeted therapy with oral agents is expected to rise in future. To date, no study has been conducted on the actual experience and process of establishing selfmanagement in elderly patients with lung cancer receiving molecularly targeted therapy with oral agents in the outpatient setting with the aim of elucidating how self-management can be practiced. 


\section{Purpose of the Study}

The purpose of this study was to elucidate the process by which elderly patients with lung cancer on molecularly targeted therapy with oral agents in an outpatient setting establish self-management and to discuss the nursing care that should be provided to these patients.

\section{Definition of Terms}

\section{Self-Management}

Referring to the definition by Knobf et al. [9], self-management was defined as "a process in which patients cope with side effects and other disadvantages in daily life in a way that suits them and spend their lives during recuperation in accordance with their preferences/wishes, utilizing the support of their family and health care providers after understanding the disease and therapy."

\section{Methods}

\subsection{Subjects}

Subjects of the study were lung cancer patients aged between 65 and 84 years who were receiving molecularly targeted therapy with oral agents in an outpatient setting at one of the two sentinel sites for cancer therapy in the Osaka and Hyogo prefectures, with an elapse of one month or longer since treatment began and who gave their informed consent to participate in the study. The researcher were informed of potential candidates by doctors and nurses of the study sites and contacted the candidates directly to ask for their participation in the study.

\subsection{Data Collection}

The researcher confirmed that candidates met the criteria for participation, such as age and time from treatment initiation. Theoretical sampling was used and data were collected from subjects who provided their consent for participation. A semi-structured interview for 30 to 60 minutes per session was held with each subject using an interview guide. The interview guide included aspects such as: "when continuing therapy, what has changed in your life, how did you feel or what did you think about the change, and have you taken any action in response to the change?" and "what support do you receive that makes it possible to continue therapy?" The interview was held during outpatient waiting time or after the visit. The interview was held in a restricted area where the patient's privacy could be protected. The interview was recorded on an IC recorder with the subject's permission. Information on basic attributes (age, sex, treatment progress, medical history, familial status, etc.) was collected from medical records to be used as additional information for data analysis. Data were collected between August 2016 and February 2017.

\subsection{Study Method}

Data analysis was conducted using Kinoshita's “Modified Grounded Theory 
Approach (M-GTA)" [10] [11]. Grounded theory is a methodology particularly suitable for research in the field of human services and for studies in which the phenomenon under study is a process. Based on the original work by Straus and Glaser, M-GTA was a development of classic grounded theory and it uses 'an analytic worksheet' for exact coding and deep interpretation. As the theory thus constructed will be additionally verified and its efficacy further established through practical application, this approach was considered to be particularly appropriate for the present study.

My study's analytic themes were "a series of processes during ongoing treatment in daily life through which the subjects understands lung cancer and its treatment, and based on this understanding, finds his/her own way of coping with side effects and other disadvantages in daily life and spends his/her life during the recuperation period based on his/her preferences/wishes." From the verbatim transcript data of the interview, concepts were extracted according to the analytical theme and analytical work sheets were created for each concept. On each work sheet, the concept name, definition, variations, and theoretical memos were entered. The concepts were interpreted from the viewpoint of the analytical theme and analytically-focused person, and in parallel with data collection, variations, opposites, and similarities were confirmed. Each concept was compared with the other concepts, and data collection and analysis were conducted until theoretical saturation was achieved.

Next, categories consisting of multiple concepts were created based on relationships between concepts. Furthermore, it was examined where within the process of self-management these concepts and categories are to be positioned and the results were presented in a results chart showing the relationships. The results chart was explained in simple language using the concepts and categories created and a storyline was developed. The process of analysis was supervised by experts who are familiar with qualitative research and oncology nursing research to ensure the dependability and relevance of analysis.

\subsection{Ethics}

This study was approved by the committee for research ethics at Osaka Medical College (No. 1934), the committee for research ethics at Hyogo University of Health Sciences (No. 16009). Every participant signed an informed consent form before participation, and they were given the opportunity to withdraw consent after the interview.

Because the participant of this study were elderly patient who under treatment, I explained the contents of my research to doctors and nurses in outpatient section, and prepared a system to deal with it immediately when the patient's condition got worse.

\section{Results}

\subsection{Participants}

Seventeen participants ( 8 males and 9 females) aged between 65 and 84 years 
gave their voluntary consent to participate in the study (Table 1 ). The average age was 75.5 ( $\mathrm{SD} \pm 6.3$ ) years. The duration of treatment ranged from 1 to 39 months (with an average duration of approximately 13 months).

All patients had some comorbidity including high blood pressure and diabetes mellitus. There were 7 participants with a history of cancer other than lung cancer, including stomach cancer and prostate cancer. The number of participants who lived with their families was 13 and the number of solo were 4 . The average time to interview was 35 minutes (21 - 65 minutes).

\subsection{Storyline}

The relationships between the categories were shown in the results diagram, and the following storyline was developed. The process of establishing self-management in elderly patients with lung cancer who are receiving molecularly targeted therapy with oral agents in an outpatient setting is as follows (Figure 1). In the following, "Bold" represents categories.

Once they were informed of the disease and started oral molecular targeted therapy, these elderly patients tried to both Accept life with cancer and Maintain activities of daily living without feeling shackled by cancer. They started to Reconsidering how to continue being themselves in daily life until life comes to an end. This constituted the basis for the participants to repeat a feedback loop of

Table 1. Characteristics of participants.

\begin{tabular}{|c|c|c|c|c|}
\hline Participant & Age & Gender & $\begin{array}{l}\text { The duration of treatment } \\
\text { (months) }\end{array}$ & Molecularly targeted drug \\
\hline A & 60 's & $\mathrm{F}$ & 8 & Gefitinib \\
\hline B & 80 's & $\mathrm{F}$ & 17 & Erlotinib (2nd line) \\
\hline $\mathrm{C}$ & 80 's & $\mathrm{F}$ & 1 & Gefitinib \\
\hline $\mathrm{D}$ & 70 's & M & 15 & Erlotinib \\
\hline E & 60 's & M & 16 & Afatinib \\
\hline $\mathrm{F}$ & 70 's & $\mathrm{F}$ & 10 & Osimertinib (2nd line) \\
\hline G & 70 's & $\mathrm{F}$ & 39 & Gefitinib \\
\hline $\mathrm{H}$ & 80 's & $\mathrm{F}$ & 1 & Gefitinib \\
\hline I & 70 's & $\mathrm{F}$ & 2 & Gefitinib \\
\hline $\mathrm{J}$ & 70 's & M & 2 & Gefitinib \\
\hline K & 60 's & M & 22 & Osimertinib (4th line) \\
\hline $\mathrm{L}$ & 80 's & M & 8 & Gefitinib \\
\hline M & 70 's & M & 29 & Erlotinib (2nd line) \\
\hline $\mathrm{N}$ & 60 's & $\mathrm{F}$ & 3 & Erlotinib \\
\hline $\mathrm{O}$ & 80 's & M & 7 & Gefitinib \\
\hline $\mathrm{P}$ & 60 's & M & 31 & Afatinib \\
\hline $\mathrm{Q}$ & 80 's & $\mathrm{F}$ & 13 & Osimertinib (2nd line) \\
\hline
\end{tabular}




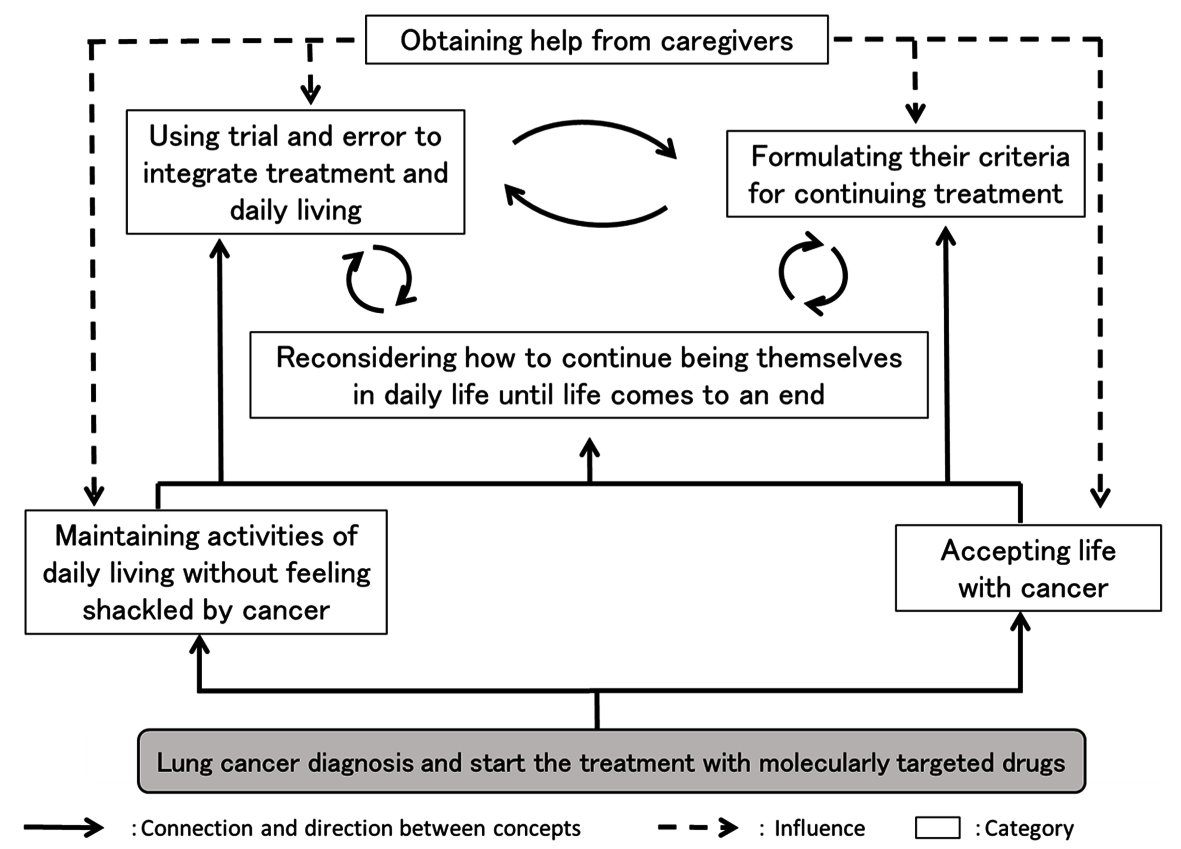

Figure 1. The process of elderly patients with lung cancer who are undergoing treatment with molecularly targeted drugs establish self-management.

Using trial and error to integrate treatment and daily living, and Formulating their criteria for continuing treatment. Through this repeated cycle, patients weighed both therapeutic and side effects, and explored ways of living their own lives. Some transitioned from Accepting life with cancer and Maintaining activities of daily living without feeling shackled by cancer directly to Using trial and error to integrate treatment and daily living, and Formulating their criteria for continuing treatment, but skipped the reconsidering phase. Obtaining help from caregivers impacted and reinforced the other four categories.

\subsection{Description of Concepts and Categories}

Nineteen concepts were generated which were organized into 6 categories according to their similarity (Table 2).

In the following, "Bold-Italic" represents concepts, "Italic" represents narratives of subjects, and subjects' ID code in round brackets ( ).

\section{1) Accepting life with cancer}

Accepting life with cancer means that the patient, though healthy so far, faces the fact of having cancer and becomes aware of death, and accepts having to live with the disease. This category consists of two concepts: Accepting the fact that they have cancer and Accepting the naturalness of having cancer.

\section{Accepting the fact that they have cancer}

"When I was diagnosed with cancer, it was already Stage III. The doctor was not sure if I would survive five years. I thought-hmmm-prognosis of lung cancer is so poor. (ID N)"

\section{Accepting the naturalness of having cancer}


Table 2. Categories and concepts that constitute self-management process.

\begin{tabular}{|c|c|}
\hline Category & Concept \\
\hline Accepting life with cancer & $\begin{array}{l}\text { Accepting the fact that they have cancer } \\
\text { Accepting the naturalness of having cancer }\end{array}$ \\
\hline $\begin{array}{l}\text { Maintaining activities } \\
\text { of daily living without } \\
\text { feeling shackled by cancer }\end{array}$ & $\begin{array}{l}\text { Exploring ways of reducing fixation on the disease } \\
\text { Improving the non-patient aspects of life in a healthy way } \\
\text { Maintaining a take-it-easy attitude } \\
\text { Continuing to be themselves as before }\end{array}$ \\
\hline $\begin{array}{l}\text { Reconsidering how to } \\
\text { continue being themselves } \\
\text { in daily life until life } \\
\text { comes to an end }\end{array}$ & $\begin{array}{l}\text { Changing their lifestyle as a preparation for getting older } \\
\text { Envisioning a future beyond treatment } \\
\text { Weighing life and medical expenses }\end{array}$ \\
\hline $\begin{array}{l}\text { Using trial and error to } \\
\text { integrate treatment } \\
\text { and daily living }\end{array}$ & $\begin{array}{l}\text { Trusting and complying with the information given } \\
\text { by health care providers } \\
\text { Devising ways to protect one's life from being unduly } \\
\text { burdened by side effects } \\
\text { Negotiating with health care providers }\end{array}$ \\
\hline $\begin{array}{l}\text { Formulating their criteria } \\
\text { for continuing treatment }\end{array}$ & $\begin{array}{l}\text { Enduring side effects to the limits of mental and } \\
\text { physical capacity } \\
\text { Establishing criteria as to what extent side effects can be tolerated } \\
\text { Continuing treatment when its effects can be felt } \\
\text { Becoming resilient and accepting side effects }\end{array}$ \\
\hline $\begin{array}{l}\text { Obtaining help } \\
\text { from caregivers }\end{array}$ & $\begin{array}{l}\text { Accepting support so treatment can continue } \\
\text { Relying on others to maintain their own lifestyle } \\
\text { Accepting moral support from family and friends }\end{array}$ \\
\hline
\end{tabular}

"I don't care much about having cancer. I think becoming ill in old age is very common. You often hear that an elderly friend or neighbor has become ill. So, it just happened to me this time. (ID B)"

2) Maintaining activities of daily living without feeling shackled by cancer

Maintaining activities of daily living without feeling shackled by cancer means that, instead of thinking all the time about having cancer, the patient tries to maintain his/her life as a person in an acceptable state of health for his/her age, by changing habits to more healthy ones or by not letting the disease become a burden. This category consists of four concepts: Exploring ways of reducing fixation on the disease, Improving the non-patient aspects of life in a healthy way, Maintaining a take-it-easy attitude, Continuing to be themselves as before.

\section{Exploring ways of reducing fixation on the disease}

"Diagnosed with cancer, the worst thing I can do is to be depressed and think about bad things only. The only thing I care about is to maintain a positive outlook, not to care about what others say, and to live a regular life. Emotional stress is not good for me. (ID F)" 
Improving the non-patient aspects of life in a healthy way

"I ask my family to let me have bananas, tomatoes and 'Natto' for my health. You know, these are good for your health. (ID H)"

\section{Maintaining a take-it-easy attitude}

"My wife tells me to quit smoking. So, these days, I do not smoke so much. I used to smoke one pack a day before, but now, I smoke only 1/2 pack a day. I'll try and quit smoking little by little. (ID )"

\section{Continuing to be themselves as before}

"Fortunately, we will have winter soon. This means, I can wear a mask to hide the eczema on my face and say it is because I have a cold. I don't need to explain to my friends about the side effects of drugs. (ID I)"

3) Reconsidering how to continue being themselves in daily life until life comes to an end

Reconsidering how to continue being themselves in daily life until life comes to an end means that patients, while continuing therapy, feel the influences of disease and treatment and recognize that an age-related decline in their abilities is occurring, but still find ways to live in the manner of their own choosing for the remainder of their lives. This category consists of three concepts: Changing their lifestyle as a preparation for getting older, Envisioning a future beyond treatment, and Weighing life and medical expenses.

\section{Changing their lifestyle as a preparation for getting older}

"My hobby is growing chrysanthemums, which is getting more difficult because I can no longer lift heavy pots. But I don't think it is good for me to give up my hobby all together. So, I decided to grow smaller plants. (ID O)"

\section{Envisioning a future beyond treatment}

"I started taking this drug right after its launch, so I cannot compare my condition with those of other patients. If there were a lot of data about drug use in other patients, the doctor could determine whether I should continue taking this drug or stop taking it. But there are no such data. (ID E)"

\section{Weighing life and medical expenses}

"To be honest, it's hard for me to pay for the expensive drug. I have to pay 8,000 yen every month and this goes on forever. (ID Q)"

4) Using trial and error to integrate treatment and daily living

Using trial and error to integrate treatment and daily living means patients embark upon a process of trial and error to make treatment part of their lives and to find out how they can spend their lives in a way that is satisfying to them. This category consists of three concepts: Trusting and complying with the information given by health care providers, Devising ways to protect their lives from being unduly burdened by side effects, and Negotiating with health care providers.

Trusting and complying with the information given by health care providers

"I am ready to strictly comply with the instructions given by my doctor. I will take the drug every day, exactly at the specified time as instructed. I completely 
trust and leave everything to my doctor. (ID M)"

Devising ways to protect their lives from being unduly burdened by side effects

"I had inflammation (paronychia) and had terrible pain in my toenail. I had pain when wearing a shoe, so I cut open the toe area of my shoe. (ID D)"

Negotiating with health care providers

"I used to take the drug every day, and then I developed eczema on my right leg. My leg was itchy so that I scratched it and caused a sore. For me, this was a very annoying event, so I asked my doctor to reduce the dose to once every other day. (ID G)"

5) Formulating their criteria for continuing treatment

Formulating their criteria for continuing treatment means that patients determine the optimum extent of treatment, considering the side effects associated with continued treatment and the burden imposed on their lives and reflecting on how to spend their lives from that point onward. This category consists of four concepts: Enduring side effects to the limits of mental and physical capacity, Establishing criteria for the extent to which they can tolerate side effects, Continuing treatment when its effects can be felt, and Becoming resilient and accepting side effects.

Enduring side effects to the limits of mental and physical capacity

"I only know the side effects that occur in me. Maybe other patients are suffering more than I do; I must not think that I am the only person who is suffering. You have to help yourself. I don't want to bother the outpatient nurses who are so busy. (ID P)"

Establishing criteria for the extent to which they can tolerate side effects

"I hope that the lung cancer can be cured. However, I think I should stop treatment if side effects become too severe. If I feel sick, I'll tell my doctor that I want to have the dose reduced. (ID B)"

\section{Continuing treatment when its effects can be felt}

"The drug was effective against my disease. Therefore, I will do my best to avoid side effects. I have heard that you have to stop using this drug if the side effects become too severe. (ID A)"

\section{Becoming resilient and accepting side effects}

"My doctor said that hair dyeing may aggravate side effects on the skin. Therefore, I use a wig to hide my white hair. However, now the wig looks so unnatural because my hair has grown long, so I do not wear the wig any more. (ID A)"

\section{6) Obtaining help from caregivers}

Obtaining help from caregivers means that patients, when diagnosed with cancer and receiving treatment, realize that they cannot live without help from others and decide to continue living while undergoing medical treatment with support from people close to them and health care providers with whom they have developed a close relationship, and in cooperation those they have created new relationships with. The category consists of three concepts: Accepting 
support so treatment can continue, Relying on others to maintain their own lifestyle, Accepting moral support from family and friends.

Accepting support so treatment can continue

"My wife was worried about me and bought supplements to compensate for nutrients and prepared meat dishes to improve my anemia. Later, my blood test results were improved. (ID L)"

\section{Relying on others to maintain their own lifestyle}

"My daughter says 'Tell me what you need. I can go shopping and bring it to you.' She is living far away from me; however, since I am sick, she often comes to see me. (IDC)"

\section{Accepting moral support from family and friends}

"My neighbors say that my wife and I get along very well. It is important to say "Thank You" to my wife. Since I have cancer, I have come to the strong realization that being thankful is important in life. (ID K)"

\section{Discussion}

\subsection{Process of Establishing Self-Management in Elderly Patients with Lung Cancer Receiving Molecularly Targeted Therapy with Oral Agents in an Outpatient Setting}

After being informed of cancer and having started molecularly targeted therapy with oral agents in an outpatient setting, elderly patients with lung cancer consider their old age and looked back on their lives and came to accept the fact that having cancer is a natural event. Compared to younger adult patients, elderly patients have often experienced other diseases before getting cancer, therefore, they find it less difficult to accept physical decline and reduced social engagement, and it has been reported that they suffer a less-pronounced reduction in QOL [12]. A study in patients with metastatic cancer also suggested that the level of depression in elderly patients is significantly lower compared to younger adult patients, and, because they have greater emotional stability and maturity, they are more able to endure sickness [13]. In the present study, too, the elderly patients with lung cancer accepted having cancer as a natural event, which demonstrates that elderly people have a way of accepting disease unique to their age group. The subjects of this study accepted living with cancer, however, they still made efforts to maintain activities of daily living without feeling shackled by cancer. By carrying on with their daily lives as before, they were making efforts to live without becoming obsessed by cancer. Elderly cancer patients do not seem to develop excessive anxiety about their condition. Rather, by focusing on how to continue being themselves, they seem to have the "strength" to face up to their therapy.

It was observed that subjects endeavored to some degree to live their own lives in the manner of their own choosing while they continued therapy and to reconsider how to continue being themselves in daily life until their lives came to an end. A previous study has shown that pride in living an independent 
life is something that gives elderly people the strength to be themselves even though they need support and care from others [14]. Living an independent life, albeit with some support, is considered to be of great relevance for elderly people who accept being old and face physical and mental decline. According to Erikson [15], loss of abilities including physical decline, reduction in autonomy, and realizing that death is inevitable are considered developmental crises unique to old age. Reflecting back on the life they have lived and coming to a balance between despair and acceptance/integration is the developmental challenge that the elderly face, Erikson says. The subjects of the present study went through various processes and adopted a trial and error approach to therapy and reconsidered how to continue being themselves in daily life until their lives came to an end. This seems to be very much an example of tackling the above developmental task of old age, i.e., achieving acceptance/integration in life.

In the stage of Reconsidering how to continue being themselves in daily life until their lives came to an end, patients were at a loss as to how they could bear the financial burden associated with prolonged therapy and the continuation of therapy without future prospects. Molecularly targeted agents are much more expensive than traditional cytotoxic anticancer drugs. Even when able to access high-cost medical care benefits, the co-payment still has a major financial impact on elderly people. As newer drugs are developed and launched, patients are told that if one drug ceases to be effective, a new one may be used. Some of the subjects of the present study, particularly those receiving prolonged therapy, conducted self-management while at the same time worrying how long they would be able to or would have to continue with untried, state-of-the-art therapy. At the same time, subjects were Formulating their criteria for continuing treatment; some subjects did not want to receive therapy with unpleasant side effects, while others were ready to do their best to continue receiving therapy. When elderly patients are to continue with therapy which is not expected to cure their lung cancer, it is important for each patient to make sure whether or not, and in what way, the therapy will benefit them for the remainder of their lives. Health care providers should help to crystallize the patient's intention and provide information in accordance with this intention; they should support patients to spend their lives in the manner of their choosing.

In order to reconsider how to continue being themselves in daily life until their lives came to an end and achieve balance between living the life they want and therapy without stress, patients were Formulating their criteria for continuing treatment. Using these criteria, a process of trial and error was used to integrate treatment into daily living. Obtaining help from caregivers influenced several aspects of the process of establishing self-management. In the present study, subjects were able to accept the fact that there are things they cannot do or understand due to reduced ADL and decline in memory. With this acceptance, they received support from others in situations where they could be helpful, and were thus able to manage their daily activities in the ways they wanted while continuing to receive therapy. This is a strength unique to elderly 
people. These observations emphasize the importance of social support for elderly cancer patients to help them maintain stability in their daily lives while continuing therapy.

Some subjects in the present study placed such great importance on the information given by their health care providers, that they continued taking the drug as instructed even when their condition had deteriorated or when enduring side effects without consulting their doctor. Results of a previous study showed that a high percentage of Japanese elderly people consider authoritative doctors to be more reliable and want doctors to maintain a confident attitude [16], suggesting that they tend to readily follow the instructions of health care providers. This attitude of elderly cancer patients, who believe that explanations and advice given by heath care providers must invariably be adhered to, may be useful in that it makes appropriate intervention by health care providers very effective. In a symptom management intervention study in elderly cancer patients on chemotherapy [17], over $90 \%$ of the subjects in the elderly patient group maintained contact with their nurse after a nurse-led intervention, and the symptoms were milder and QOL significantly higher in the elderly patient group compared to the younger adult group. Appropriate support should be provided to elderly cancer patients so they can manage their symptoms effectively and live their own lives in the manner of their own choosing. Nurses providing support should keep in mind that elderly patients tend to be very serious about undergoing therapy, sometimes making them excessively compliant or reserved with health care providers so that they may be hesitant to complain of pain and discomfort.

\subsection{Suggestions for Nursing Practice}

For elderly patients with lung cancer, such as the subjects of the present study, self-management is not limited to management of symptoms, but is rather an ongoing process with the aim of enabling patients to live their own lives in the manner of their choosing while accepting the disease and the changes due to old age. Based on this perception, nurses should provide the following support to elderly patients with lung cancer:

1) Nurses should help patients to clarify their thinking about living their own lives in the manner of their own choosing and about how to spend the rest of their lives.

2) Nurses should provide information regarding therapeutic options and treatment of side effects to help patients live their own lives in the manner of their own choosing.

3) Nurses should engage in close communication with doctors and other health care providers seeking to promote continuation of therapy in accordance with the patient's wishes.

To preserve QOL in cancer patients receiving treatment, it is essential to manage treatment-related side effects and disease-related symptoms. Especially in the case of elderly patients, nurses should always respect the patient's beliefs 
and provide support based on how symptoms affect the patient's daily activities and the significance these activities have for the patient. This means nurses have to provide support in symptom management so that patients can live their lives in the manner of their own choosing and help them understand not only their symptoms but also the situation they are facing, including the conditions of daily living.

The results obtained in the present study and previous studies conducted in elderly patients, i.e., that elderly patients tend to trust and comply with the in formation given by health care providers and devise ways to protect their lives from being unduly burdened by side effects suggest that elderly patients with lung cancer will be able to exercise their unique strengths when they communicate well with health care providers and discuss the most appropriate direction of therapy and methods for dealing with side effects. It has been reported that most elderly patients have difficulties communicating with health care providers due to various age-related factors [18]. The subjects of the present study, however, were able to negotiate with their doctors with the help of family members or consulted doctors with the help of a health care provider with whom they shared a closer relationship such as a nurse. Nurses need to acquire the knowledge and skills that allow them to appropriately communicate with elderly patients and to help them to maintain smooth collaboration between themselves and those around them.

The verbal responses of subjects revealed that elderly patients with lung cancer who are in need of various forms of support are left almost without any support in the outpatient setting. The results demonstrate the need for devising a practical method of nursing care in order to provide adequate help and information to elderly patients in accordance with how they wish to live their lives and to make it possible for them to manifest their strengths and to spend their lives during recuperation in the manner of their own choosing.

\subsection{Limitations and Future Issues}

1) The results of the present study demonstrated the necessity of providing support to promote self-management suited to the characteristics of each patient. Therefore, a systematic program to support self-management and appropriate criteria for evaluating the effectiveness of interventions must be devised and its feasibility studied to make it possible to continuously support elderly patients with lung cancer receiving molecularly targeted therapy with oral agents.

2) The subjects of the present study had relatively well-preserved ADL functioning, and most of them had no major cognitive impairment. Further studies are necessary in subjects with various age-related issues so that the results of these studies can be applied to a wider range of patients.

\section{Conclusions}

In order to establish self-management in elderly patients with lung cancer re- 
ceiving molecularly targeted therapy with oral agents in the outpatient setting, the following six categories were created: Accepting life with cancer, Maintaining activities of daily living without feeling shackled by cancer, Reconsidering how to continue being themselves in daily life until life comes to an end, Using trial and error to integrate treatment and daily living, Formulating their criteria for continuing treatment, and Obtaining help from caregivers.

In the process of establishing self-management in these patients, nurses have to assess for each individual the patient's way of life and aspirations and provide support in symptom management so that they can live their lives in the manner of their own choosing. Support is necessary to help self-management of not only symptoms but of daily living itself. The findings of this study also suggest that patients should be given support to help them establish and maintain smooth collaboration between themselves and the people around them.

\section{Acknowledgements}

I would like to express my gratitude to all participants and hospital staff for their cooperation in this research. And I would like to give thanks to Prof. Suzuki who provided considered feedback and valuable comments. I am also indebted to Prof. Tomari, Prof. Tanaka, and Prof. Yamauchi who provided technical help and sincere encouragement.

\section{Declaration of a Conflict of Interests}

I have no potential conflicts of interest with respect to the research, authorship, and/or publication of this article.

\section{Funding}

I received the following financial support for the research, authorship, and/or publication of this article: This work was supported by JSPS KAKENHI Grant Number JP16K20782.

\section{References}

[1] Center for Cancer Control and Information Services Japan. (In Japanese) http://ganjoho.jp/reg_stat/statistics/stat/summary.html

[2] The Japan Lung Cancer Society (2016) Lung Cancer Clinical Practice Guidelines Based on EBM Method 2016 Edition, Kanehara Shuppan. Tokyo, 122-123, 125. (In Japanese)

[3] Bezjak, A., Tu, D., Seymour, L., Clark, G., Trajkovic, A., Zukin, M., et al. (2006) Symptom Improvement in Lung Cancer Patients Treated with Erlotinib: Quality of Life Analysis of the National Cancer Institute of Canada Clinical Trials Group Study BR.21. Journal of Clinical Oncology, 24, 3831-3837. https://doi.org/10.1200/JCO.2006.05.8073

[4] Hayashi, T., Kitaoka, T., Uto, N., Hiraga, H., Kondo, G., Otsubo, Y., et al. (2006) A Multicenter Survey on the Proper Use and Dosage of Erlotinib in Japanese Patients 
with Non-Small Cell Lung Cancer. Journal of Japanese Society of Hospital Pharmacists, 49, 539-543. (In Japanese)

[5] Kudoh, S., Kato, H., Nishiwaki, Y., Fukuoka, M., Nakata, K., Ichinose, Y., et al. (2008) Interstitial Lung Disease in Japanese Patients with Lung Cancer: A Cohort and Nested Case-Control Study. American Journal of Respiratry and Critical Care Medicine, 177, 1348-1357. https://doi.org/10.1164/rccm.200710-1501OC

[6] Komatsu, H., Yagasaki, K. and Yoshimura, K. (2014) Current Nursing Practice for Patients on Oral Chemotherapy: A Multicenter Survey in Japan. BMC Research Notes, 7, 259. https://doi.org/10.1186/1756-0500-7-259

[7] Jooste, V., Woronoff, A.S., Quipourt, V., Bouvier, A.M., Mercier, M., Fournier, E., Jooste, V., Woronoff, A.S., et al. (2016) Health-Related Quality of Life Is a Prognostic Factor for Survival in Older Patients after Colorectal Cancer Diagnosis: A Population-Based Study. Digestive and Liver Disease, 48, 87-93. https://doi.org/10.1016/j.dld.2015.09.006

[8] Fukawa, A. (2017) Quality of Life in Older Cancer Patients Who Receiving Chemotherapy : A Literature Review. Journal of Japanese Society of Cancer Nursing, 31, 76-81. (In Japanese)

[9] Knobf, M.T., Cooley, M.E., Duffy, S., et al. Oncology Nursing Society 2014-2018 Research Agenda.

https://www.ons.org/sites/default/files/2014-2018\%20ONS\%20Research\%20Agenda $\underline{\text { pdf }}$

[10] Kinoshita, Y. (2003) Practice of Grounded Theory Approach, Tokyo, Koubundou. (In Japanese)

[11] Kinoshita, Y. (2007) The Live Lecture on M-GTA: The Practical Qualitative Research Methodology, All Modified Grounded Theory Approach, Tokyo, Koubundou. (In Japanese)

[12] Quinten, C., Coens, C., Ghislain, I., Zikos, E., Sprangers, M.A., Ringash, J., et al. (2015) The Effects of Age on Health-Related Quality of Life in Cancer Populations: A Pooled Analysis of Randomized Controlled Trials using the European Organisation for Research and Treatment of Cancer (EORTC) QLQ-C30 Involving 6024 Cancer Patients. European Journal of Cancer, 51, 2808-2819.

https://doi.org/10.1016/j.ejca.2015.08.027

[13] Lo, C., Lin, J., Gagliese, L., Zimmermann, C., Mikulincer, M. and Rodin, G. (2010) Age and Depression in Patients with Metastatic Cancer: The Protective Effects of Attachment Security and Spiritual Wellbeing. Ageing and Society, 30, 325-336. https://doi.org/10.1017/S0144686X09990201

[14] Yoshio, C., Mimura, N. and Tomita, M. (2010) The Constructive Consepts of Zest for Living among Older People Who Require Nursing Care. Journal of Japan Academy of Home Care, 14, 31-38. (In Japanese)

[15] Erikson, E. and Erikson, J. (1998) The Life Cycle Completed. Extended Version Edition, W. W. Norton \& Company, New York.

[16] Nishigaki, E. (2008) Typology and Characterisitics of Japanese Patients from the Perspective of Patients-Physician Trust. The Japanese Journal of Health Psychology, 21, 1-9. (In Japanese) https://doi.org/10.11560/jahp.21.1_1

[17] Mkanta, W.N., Chumbler, N.R., Richardson, L.C. and Kobb, R.F. (2007) Age-Related Differences in Quality of Life in Cancer Patients: A Pilot Study of a Cancer Care Coordination/Home-Telehealth Program. Cancer Nursing, 30, 434-440. https://doi.org/10.1097/01.NCC.0000300167.80466.91 
[18] Van Weert, J.C., Bolle, S., van Dulmen, S. and Jansen, J. (2013) Older Cancer Patients' Information and Communication Needs: What They Want Is What They Get? Patient Education and Counseling, 92, 388-397.

https://doi.org/10.1016/j.pec.2013.03.011 